\title{
GEOMETRIC DETERMINATION OF THE POLES OF HIGHEST AND SECOND HIGHEST ORDER OF HODGE AND MOTIVIC ZETA FUNCTIONS
}

\author{
B. RODRIGUES*
}

\begin{abstract}
To any $f \in \mathbb{C}\left[x_{1}, \ldots, x_{n}\right] \backslash \mathbb{C}$ with $f(0)=0$ one can associate the motivic zeta function. Another interesting singularity invariant of $f^{-1}\{0\}$ is the zeta function on the level of Hodge polynomials, which is actually just a specialization of the motivic one. In this paper we generalize for the Hodge zeta function the result of Veys which provided for $n=2$ a complete geometric determination of the poles. More precisely we give in arbitrary dimension a complete geometric determination of the poles of order $n-1$ and $n$. We also show how to obtain the same results for the motivic zeta function.
\end{abstract}

\section{Introduction}

0.1. Let $f \in \mathbb{C}\left[x_{1}, \ldots, x_{n}\right] \backslash \mathbb{C}$ with $f(0)=0$ and let $h: X \rightarrow U$ be an embedded resolution of $f^{-1}\{0\}$ in the germ $\left(\mathbb{A}_{\mathbb{C}}^{n}, 0\right)$ of $\mathbb{A}_{\mathbb{C}}^{n}$ at the origin, where $U$ is a Zariski open subset of $\mathbb{A}_{\mathbb{C}}^{n}$ containing the origin. Denote by $E_{i}, i \in S$, the irreducible components of $h^{-1}\left(f^{-1}\{0\}\right)$ and set $E_{I}^{\circ}:=$ $\left(\bigcap_{i \in I} E_{i}\right) \backslash\left(\bigcup_{l \notin I} E_{l}\right)$ for $I \subseteq S$. So $X$ is the disjoint union of the locally closed strata $E_{I}^{\circ}, I \subseteq S$. For $i \in S$ we also denote by $N_{i}$ and $\nu_{i}-1$ the multiplicities of $E_{i}$ in the divisor on $X$ of $f \circ h$ and $h^{*}\left(d x_{1} \wedge \cdots \wedge d x_{n}\right)$, respectively.

To $f$ one associates the (local) Hodge zeta function $Z_{\mathrm{Hod}}(f, s)$, which is an interesting singularity invariant of the germ of $f^{-1}\{0\}$ at the origin. It is defined as follows in terms of the embedded resolution $h$ :

$$
Z_{\mathrm{Hod}}(f, s):=\sum_{I \subseteq S} H\left(E_{I}^{\circ} \cap h^{-1}\{0\}\right) \prod_{i \in I} \frac{u v-1}{(u v)^{\nu_{i}+s N_{i}}-1} \in \mathbb{Q}(u, v)(T),
$$

where $H(\cdot)$ denotes the Hodge polynomial. For each variety $V$ this Hodge polynomial $H(V)$ is a polynomial over $\mathbb{Z}$ in two variables $u$ and $v$, see (1.3).

Received July 10, 2003.

2000 Mathematics Subject Classification: 14B05 14E15 14J17 (32S45).

${ }^{*}$ Postdoctoral Fellow of the Fund for Scientific Research - Flanders (Belgium). 
The expression above, which should be considered as a rational function in the variable $T=(u v)^{-s}$, does not depend on the chosen resolution. This is an immediate consequence of the fact that it is a specialization of the wellknown motivic zeta function, which is intrinsically defined in [4] by Denef and Loeser using motivic integration. In the same paper also the following formula in terms of the resolution $h$ is derived:

$$
Z_{\mathrm{mot}}(f, s)=\sum_{I \subseteq S}\left[E_{I}^{\circ} \cap h^{-1}\{0\}\right] \prod_{i \in I} \frac{\mathbb{L}-1}{\mathbb{L}^{\nu_{i}+s N_{i}}-1} .
$$

(Actually Denef and Loeser obtained that $\mathbb{L}^{n} Z_{\operatorname{mot}}(f, s)$ equals the righthand side of the equality above; but sometimes the definition is rescaled to eliminate the factor $\mathbb{L}^{n}$.) Here we denote by $[\cdot]$ the class in the Grothendieck ring of algebraic varieties over $\mathbb{C}$ and by $\mathcal{M}_{\mathbb{C}}$ the localization of this ring with respect to $\mathbb{L}:=\left[\mathbb{A}_{\mathbb{C}}^{1}\right]$; see (1.3). Then this zeta function lives in a localization of the polynomial ring $\mathcal{M}_{\mathbb{C}}\left[\mathbb{L}^{-s}\right]$.

Interesting about the Hodge zeta function $Z_{\text {Hod }}(f, s)$ is for example that almost all propositions for the motivic zeta function also hold already for $Z_{\text {Hod }}(f, s)$; thus, for a lot of purposes the level of Hodge polynomials turns out to be fine enough already. And on this level we at least work over an integral domain; recall in this context the result of Poonen [9] stating that the Grothendieck ring of varieties is not an integral domain.

0.2. We are especially interested in the poles of these zeta functions. Here we say that a rational number $q$ is a pole of order $d$ of $Z_{\text {Hod }}(f, s)$ if $(u v)^{-q}$ is, considering $Z_{\mathrm{Hod}}(f, s)$ as a rational function in the variable $T=(u v)^{-s}$. For the notion of a pole of the motivic zeta function, see (3.1).

Clearly the only possible poles are the elements of the set $\left\{-\nu_{i} / N_{i} \mid\right.$ $i \in S\}$. Hence we will simply call them the candidate poles. It is quite interesting to note that the absolute value $\min _{i \in S}\left\{\nu_{i} / N_{i}\right\}$ of the largest candidate pole is in fact just the $\log$ canonical threshold of $f$ at 0 (and is thus independent of the chosen resolution), see [6, Section 8]. The whole set $\left\{-\nu_{i} / N_{i} \mid i \in S\right\}$ is of course not an invariant of $f$, but its subset consisting of the poles of $Z_{\text {Hod }}(f, s)$ is. Philosophically the poles of these zeta functions are induced by "important" components $E_{i}$, which occur in every resolution.

Furthermore, these poles are related to eigenvalues of the local monodromy of $f: X \rightarrow \mathbb{A}_{\mathbb{C}}^{n}$ by the remarkable Monodromy Conjecture. This conjecture states that for any pole $s_{\circ}$ of $Z_{\mathrm{Hod}}(f, s)$ the complex number 
$e^{2 \pi i s}$ is an eigenvalue of the local monodromy of $f$ at some point of $f^{-1}\{0\}$. For $n=2$ this conjecture is already proved; see [7] or [10]. For $n \geq 3$ it is still open, but there is a lot of evidence for it; see for example [2], [8], [11] and $[13]$.

0.3. It is an important and difficult question whether or not one can decide "geometrically" when a given candidate pole $-\nu_{i} / N_{i}, i \in S$, is a pole. In the case of two variables Veys gave a positive answer to this question, proving the following complete geometric determination of the poles.

ThEOREM 0.3.1. ([14, Theorem 4.3]) Let $n=2$ and let $h$ be the minimal embedded resolution of $f^{-1}\{0\}$ in $\left(\mathbb{A}_{\mathbb{C}}^{2}, 0\right)$. Then a rational number $s_{\circ}$ is a pole of $Z_{\mathrm{Hod}}(f, s)$ if and only if $s_{\circ}=-1 / N_{i}$ for some irreducible component $E_{i}$ of the strict transform of $f^{-1}\{0\}$ or $s_{\circ}=-\nu_{i} / N_{i}$ for some exceptional curve $E_{i}$ intersecting at least three times other components.

In fact Veys proved this result for the so-called topological zeta function, see [3] for the definition of this function; but one can easily check that this immediately implies Theorem 0.3.1. The condition on the resolution $h$ of being minimal may as well be dropped, at least when we replace "intersecting at least three times other components" by "intersecting at least three times other components $E_{j}$ with $\nu_{j}-\frac{\nu_{i}}{N_{i}} N_{j} \neq 1$ ".

Recall the formula of A'Campo [1, Theorem 3]. It states that the alternating product of all characteristic polynomials of the local monodromy of $f$ at 0 is equal to

$$
\prod_{i \in S_{e}}\left(1-t^{N_{i}}\right)^{-\chi\left(E_{i}^{\circ}\right)}
$$

where $\chi(\cdot)$ denotes the topological Euler characteristic and $S_{e}$ is the subset of $S$ selecting precisely the indices $i$ for which the corresponding variety $E_{i}$ is exceptional. Relating this fact with the Monodromy Conjecture it is not too surprising that precisely the exceptional (rational) curves $E_{i}$ intersecting at least three times other components claim a special place in Theorem 0.3.1, as they are precisely the ones satisfying $-\chi\left(E_{i}^{\circ}\right)>0$.

One can associate similar zeta functions to a nonconstant regular function $f$ on a normal surface germ $(Y, 0)$, see [16]. Also in this more general situation a complete geometric determination of the poles has been proved.

TheOREm 0.3.2. ([12, Theorem 3.4]) Let $f$ be a nonconstant regular function on a normal surface germ $(Y, 0)$, satisfying $f(0)=0$. Let $h$ : 
$X \rightarrow Y$ be any embedded resolution of $f^{-1}\{0\}$ in $(Y, 0)$, and take $s_{\circ} \in \mathbb{Q}$. Then $s_{\circ}$ is a pole of $Z_{\mathrm{Hod}}(f, s)$ if and only if

(i) $s_{\circ}=-1 / N_{i}$ for some irreducible component $E_{i}$ of the strict transform of $f^{-1}\{0\}$, or

(ii) $s_{\circ}=-\nu_{i} / N_{i}$ for some rational exceptional curve $E_{i}$ intersecting at least three times other components $E_{j}$ with $\nu_{j}-\frac{\nu_{i}}{N_{i}} N_{j} \neq 1$, or

(iii) $s_{\circ}=-\nu_{i} / N_{i}$ for a cycle of rational exceptional curves $E_{i}$, or

(iv) $s_{\circ}=-\nu_{i} / N_{i}$ for some non-rational exceptional curve $E_{i}$.

0.4. Remark that the above theorems actually give a statement about the poles of order 1 and 2. In this paper we will prove the following remarkable generalization for $n=3$, concerning the poles of order $n-1=2$ and $n=3$.

Theorem 0.4.1. Let $f \in \mathbb{C}\left[x_{1}, x_{2}, x_{3}\right] \backslash \mathbb{C}$ and let $s_{\circ} \in \mathbb{Q}$. Fix a "good" embedded resolution $h: X \rightarrow U$ of $f^{-1}\{0\}$ in $\left(\mathbb{A}_{\mathbb{C}}^{3}, 0\right)$. Let $F_{1}, \ldots, F_{r}$ be exactly the irreducible components with $h\left(F_{i}\right)=\{0\}$ of all the curves $E_{\{j, k\}}$ satisfying $s_{\circ}=-\nu_{j} / N_{j}=-\nu_{k} / N_{k}$. Then $s_{\circ}$ is a pole of $Z_{\mathrm{Hod}}(f, s)$ of order 2 or 3 if and only if

(i) there is a special point for $s_{\circ}$, or

(ii) there is a non-rational curve $F_{i}$, or

(iii) there is a rational curve $F_{i}$ intersecting at least three times other components $E_{j}$ with $\nu_{j}+s_{\circ} N_{j} \neq 1$, or

(iv) there is a cycle of rational curves $F_{i}$.

A "good" embedded resolution is defined in Subsection 1.2. For the notion of special point, see Definition 2.4.1.

We will also obtain a generalization of Theorem 0.4.1 for arbitrary dimension $n \geq 3$. For the time being one can already note that for $n=2$ a special point can be interpreted as an intersection point of an irreducible component $E_{i}$ of the strict transform of $f^{-1}\{0\}$ satisfying $s_{\circ}=-\nu_{i} / N_{i}$ with an exceptional curve $E_{j}$ satisfying $s_{\circ} \neq-\nu_{j} / N_{j}$. With this interpretation our result will also hold for $n=2$ (except in the case of a node), yielding a statement equivalent to Theorem 0.3.1; see Remark 2.8.2 for more details.

The results of the present paper also hold for the motivic zeta function $Z_{\operatorname{mot}}(f, s)$. 


\section{§1. Preliminaries}

1.1. Let $f \in \mathbb{C}\left[x_{1}, \ldots, x_{n}\right] \backslash \mathbb{C}$ with $f(0)=0$ and let $h: X \rightarrow U$ be an embedded resolution of $f^{-1}\{0\}$ in the germ $\left(\mathbb{A}_{\mathbb{C}}^{n}, 0\right)$. By this we mean that $h$ is a proper birational morphism from a nonsingular variety $X$ to a Zariski open subset $U$ of $\mathbb{A}_{\mathbb{C}}^{n}$ containing the origin such that the restriction $h: X \backslash$ $h^{-1}\left(f^{-1}\{0\}\right) \rightarrow U \backslash f^{-1}\{0\}$ is an isomorphism and $h^{-1}\left(f^{-1}\{0\}\right)$ has normal crossings in $X$. In particular the irreducible components of $h^{-1}\left(f^{-1}\{0\}\right)$ are nonsingular hypersurfaces. An embedded resolution always exists in characteristic zero by Hironaka [5]. Recall that a reduced hypersurface $E$ of $X$ has normal crossings if for all $x \in X$ there exists a regular system of parameters $t_{1}, \ldots, t_{n}$ in the local ring $O_{X, x}$ of $X$ at $x$ such that the ideal in $O_{X, x}$ of each irreducible component of $E$ containing $x$ is generated by one of the $t_{i}$.

1.2. Denote by $E_{i}, i \in S$, the (reduced) irreducible components of $h^{-1}\left(f^{-1}\{0\}\right)$. In fact, since we consider only a neighborhood of the origin $0 \in \mathbb{A}_{\mathbb{C}}^{n}$, we may as well assume that $E_{i} \cap h^{-1}\{0\} \neq \emptyset$ for each $i \in S$.

We say that the resolution $h: X \rightarrow U$ is a good embedded resolution if $h^{-1}\{0\}=\bigcup_{j \in J} E_{j}$ for some subset $J$ of $S$. One easily sees that such a good embedded resolution always exists. Indeed, we only have to start the resolution process by blowing up at the origin of $\mathbb{A}_{\mathbb{C}}^{n}$, whatever the situation might be.

Let $N_{i}$ and $\nu_{i}-1$ be the multiplicities of $E_{i}$ in the divisor on $X$ of $f \circ h$ and $h^{*}\left(d x_{1} \wedge \cdots \wedge d x_{n}\right)$, respectively. We call $\left(\nu_{i}, N_{i}\right)$ the numerical data of $E_{i}$. Although we denote by definition the irreducible components of $h^{-1}\left(f^{-1}\{0\}\right)$ by $E_{i}$, we will sometimes denote them freely by $E, E_{i}^{(j)}$, et cetera, in which case we write $(\nu, N),\left(\nu_{i}^{(j)}, N_{i}^{(j)}\right)$, et cetera, for the corresponding numerical data. For $i \in S$ and $I \subseteq S$ we denote

$$
E_{i}^{\circ}:=E_{i} \backslash \bigcup_{j \neq i} E_{j}, \quad E_{I}:=\bigcap_{i \in I} E_{i} \quad \text { and } \quad E_{I}^{\circ}:=E_{I} \backslash \bigcup_{j \in S \backslash I} E_{j} .
$$

In particular when $I=\emptyset$, we have $E_{\emptyset}=X$. Remark that $X$ is the disjoint union of the $E_{I}^{\circ}$. We obviously have for a subset $I$ of $S$ either that $E_{I}=\emptyset$ or that each irreducible component of $E_{I}$ has dimension $n-|I|$.

Note that the properness of $h$ implies that $h^{-1}\{0\}$ is complete. Hence, $h^{-1}\left(f^{-1}\{0\}\right)$ being closed in $X$, all the nonempty intersections $E_{I} \cap h^{-1}\{0\}$, with $I \subseteq S$, are complete. 
The following result of Veys will be an important tool in the proof of our geometric determination in Section 2.

Lemma 1.2.1. ([15, Example and Remark after Proposition 4.3]) Let $f \in \mathbb{C}\left[x_{1}, \ldots, x_{n}\right]$ and $s_{\circ} \in \mathbb{Q}$. Let $I \subseteq S$ such that $E_{I} \neq \emptyset,|I|=n-1$ (or equivalently $\operatorname{dim} E_{I}=1$ ) and $s_{\circ}=-\nu_{i} / N_{i}$ for $i \in I$. Consider an irreducible component $F$ of $E_{I}$ with $h(F)=\{0\}$. Let $E_{j}, j=1, \ldots, r$, be exactly the irreducible components of $h^{-1}\left(f^{-1}\{0\}\right)$ which intersect $F$ (but do not contain it). Then

$$
\sum_{j=1}^{r} m_{j}\left(\nu_{j}+s_{\circ} N_{j}-1\right)=2 g(F)-2
$$

where $m_{j}$ is the number of intersection points of $F$ and $E_{j}$.

1.3. The zeta function on the level of Hodge polynomials associated to $f$, or simply, the Hodge zeta function associated to $f$, is defined in terms of an embedded resolution $h$ as

$$
Z_{\mathrm{Hod}}(f, s):=\sum_{I \subseteq S} H\left(E_{I}^{\circ} \cap h^{-1}\{0\}\right) \prod_{i \in I} \frac{u v-1}{(u v)^{\nu_{i}+s N_{i}}-1} \in \mathbb{Q}(u, v)(T),
$$

where we consider $(u v)^{-s}$ as a variable $T$ and where $H(\cdot)$ denotes the Hodge polynomial. For a variety $V$ this Hodge polynomial is given by $H(V):=$ $\sum_{p, q} e^{p, q}(V) u^{p} v^{q} \in \mathbb{Z}[u, v]$; here $e^{p, q}(V)=\sum_{i \geq 0}(-1)^{i} h^{p, q}\left(H_{c}^{i}(V, \mathbb{C})\right)$ with $h^{p, q}\left(H_{c}^{i}(V, \mathbb{C})\right)$ the rank of the $(p, q)$-Hodge component of the $i$-th cohomology group with compact support of $V$.

The results of this paper will also apply to the motivic zeta function. In order to give the definition we first describe the Grothendieck ring of complex varieties. In this context a variety is not necessarily irreducible, it is a reduced separated scheme of finite type over $\mathbb{C}$. We denote by $K_{0}\left(\operatorname{Var}_{\mathbb{C}}\right)$ the Grothendieck group (or ring) of complex varieties. It is the abelian group generated by the symbols $[X]$, for $X$ a complex variety, with the relations $[X]=[Y]$ if $X$ and $Y$ are isomorphic as complex varieties, and $[X]=[Y]+[X \backslash Y]$ if $Y$ is Zariski closed in $X$. There is a natural ring structure on $K_{0}\left(\operatorname{Var}_{\mathbb{C}}\right)$, the product of $[X]$ and $[Y]$ being equal to $\left[X \times_{\mathbb{C}} Y\right]$. We set $\mathbb{L}:=\left[\mathbb{A}_{\mathbb{C}}^{1}\right]$ and denote by $\mathcal{M}_{\mathbb{C}}$ the ring obtained from $K_{0}\left(\operatorname{Var}_{\mathbb{C}}\right)$ by inverting $\mathbb{L}$. 
The motivic zeta function associated to $f$ can be defined in terms of an embedded resolution $h$ as follows:

$$
Z_{\mathrm{mot}}(f, s):=\sum_{I \subseteq S}\left[E_{I}^{\circ} \cap h^{-1}\{0\}\right] \prod_{i \in I} \frac{\mathbb{L}-1}{\mathbb{L}^{\nu_{i}+s N_{i}}-1}
$$

where [ $\cdot$ denotes the class in $\mathcal{M}_{\mathbb{C}}$ and where the expression $\mathbb{L}^{-s}$ should be considered as a variable.

The remarkable fact that the defining expression for $Z_{\text {mot }}(f, s)$ does not depend on the chosen resolution follows from [4, Theorem 2.2.1]. Hence the same is true for $Z_{\mathrm{Hod}}(f, s)$ since it can be obtained as a specialization of the motivic zeta function, heuristically by replacing $[\cdot]$ by $H(\cdot)$, thus by taking Hodge polynomials.

1.4. We are interested in the poles of these zeta functions. For the Hodge zeta function the notion of pole is rather clear: we say that a rational number $q$ is a pole of order $d$ if $(u v)^{-q}$ is, considering $Z_{\mathrm{Hod}}(f, s)$ as a rational function in the variable $T=(u v)^{-s}$. For the motivic zeta function we use the definition of a pole introduced in [12]. In (3.1) we will give a very brief sketch of this subtle notion.

It is easily seen that all poles of our zeta functions are of the form $-\nu_{i} / N_{i}$ for some $i \in S$. Therefore we call the rational numbers $-\nu_{i} / N_{i}, i \in$ $S$, the candidate poles. Moreover, we will say that $-\nu_{i} / N_{i}$ is the candidate pole induced by the irreducible component $E_{i}$ of $h^{-1}\left(f^{-1}\{0\}\right)$.

1.5. Note. In what follows we will often suppose, without explicitly mentioning it anymore, that a polynomial $f \in \mathbb{C}\left[x_{1}, \ldots, x_{n}\right] \backslash \mathbb{C}$ and an embedded resolution $h: X \rightarrow U$ of $f^{-1}\{0\}$ in $\left(\mathbb{A}_{\mathbb{C}}^{n}, 0\right)$ are given. We will also always assume that $f(0)=0$ and that $E_{i} \cap h^{-1}\{0\} \neq \emptyset$ for each $i \in S$; recall (1.2).

\section{§2. Geometric determination of the poles of highest and second highest order of the Hodge zeta function}

2.1. For simplicity of notation we will first treat the case of surfaces, i.e., the case $n=3$. Let us recall the formula of the Hodge zeta function in 
terms of an embedded resolution $h$ :

$$
\begin{aligned}
Z_{\text {Hod }}(f, s)= & \sum_{i \in S} H\left(E_{i}^{\circ} \cap h^{-1}\{0\}\right) \frac{u v-1}{(u v)^{\nu_{i}+s N_{i}}-1} \\
& +\sum_{\{i, j\} \subseteq S} H\left(E_{\{i, j\}}^{\circ} \cap h^{-1}\{0\}\right) \prod_{l \in\{i, j\}} \frac{u v-1}{(u v)^{\nu_{l}+s N_{l}}-1} \\
& +\sum_{\{i, j, k\} \subseteq S} H\left(E_{\{i, j, k\}} \cap h^{-1}\{0\}\right) \prod_{l \in\{i, j, k\}} \frac{u v-1}{(u v)^{\nu_{l}+s N_{l}}-1},
\end{aligned}
$$

where we use the notation of Section 1. To start the study of the poles of this function we also recall that for this purpose we consider $Z_{\text {Hod }}(f, s)$ as a rational function in $T=(u v)^{-s}$, and that we call $-\nu / N$ a pole of order $d$ if $(u v)^{\nu / N}$ is, see (1.4). Furthermore, we see that

$$
\begin{aligned}
& \frac{1}{(u v)^{\nu+s N}-1} \\
& =\frac{-T^{N}}{\left(T-(u v)^{\nu / N}\right)\left(T^{N-1}+T^{N-2}(u v)^{\nu / N}+\cdots+(u v)^{(N-1) \nu / N}\right)},
\end{aligned}
$$

and hence that $-\nu / N$ is a pole of order 1 of $1 /\left((u v)^{\nu+s N}-1\right)$.

2.2. So in order to have a candidate pole $s_{\circ}$ of order 3 we need three components $E_{1}, E_{2}$ and $E_{3}$ such that $\left(E_{1} \cap E_{2} \cap E_{3}\right) \cap h^{-1}\{0\} \neq \emptyset$ and $s_{\circ}=-\nu_{1} / N_{1}=-\nu_{2} / N_{2}=-\nu_{3} / N_{3}$. On the other hand, as soon as there is a trio of components satisfying those two conditions, $s_{\circ}$ will be a pole. Indeed, suppose that $I_{i}, i=1, \ldots, r$, are exactly the subsets of $S$ satisfying $\left|I_{i}\right|=3, E_{I_{i}} \cap h^{-1}\{0\} \neq \emptyset$ and $s_{\circ}=-\nu_{j} / N_{j}$ for $j \in I_{i}$. Since

$$
H\left(E_{I_{i}} \cap h^{-1}\{0\}\right)=\operatorname{card}\left(E_{I_{i}} \cap h^{-1}\{0\}\right),
$$

we see that $s_{\circ}$ is a pole of order 3 unless

$$
\begin{aligned}
0 & =\sum_{i=1}^{r} \operatorname{card}\left(E_{I_{i}} \cap h^{-1}\{0\}\right) \prod_{j \in I_{i}} \frac{-(u v-1)(u v)^{\nu_{j}}}{N_{j}(u v)^{\left(N_{j}-1\right) \nu_{j} / N_{j}}} \\
& =\sum_{i=1}^{r} \operatorname{card}\left(E_{I_{i}} \cap h^{-1}\{0\}\right) \frac{(1-u v)^{3}}{(u v)^{3 s_{\circ}} \prod_{j \in I_{i}} N_{j}} \\
& =\frac{(1-u v)^{3}}{(u v)^{3 s_{\circ}}}\left(\sum_{i=1}^{r} \operatorname{card}\left(E_{I_{i}} \cap h^{-1}\{0\}\right) /\left(\prod_{j \in I_{i}} N_{j}\right)\right) .
\end{aligned}
$$


But clearly the latter expression is different from zero, which implies the following result.

Proposition 2.3. Let $f \in \mathbb{C}\left[x_{1}, x_{2}, x_{3}\right] \backslash \mathbb{C}$ and let $s_{\circ} \in \mathbb{Q}$. Fix an embedded resolution $h: X \rightarrow U$ of $f^{-1}\{0\}$ in $\left(\mathbb{A}_{\mathbb{C}}^{3}, 0\right)$ and use the notation of Section 1. Then $s_{\circ}$ is a pole of $Z_{\mathrm{Hod}}(f, s)$ of order 3 if and only if there exist three components $E_{1}, E_{2}$ and $E_{3}$ of $h^{-1}\left(f^{-1}\{0\}\right)$ satisfying $\left(E_{1} \cap E_{2} \cap\right.$ $\left.E_{3}\right) \cap h^{-1}\{0\} \neq \emptyset$ and $s_{\circ}=-\nu_{1} / N_{1}=-\nu_{2} / N_{2}=-\nu_{3} / N_{3}$.

2.4. We now proceed to the poles of order 2. To make life a little bit easier, we introduce the following terminology.

Definition 2.4.1. When $h: X \rightarrow U$ is an embedded resolution of $f^{-1}\{0\}$ in $\left(\mathbb{A}_{\mathbb{C}}^{3}, 0\right)$ for some given polynomial $f$ and when $s_{\circ}$ is a given rational number, then we call a point $P \in h^{-1}\{0\}$ a special point for $s_{\circ}$ if $P$ belongs to an irreducible component $F$ of the intersection of two components $E_{1}$ and $E_{2}$ of $h^{-1}\left(f^{-1}\{0\}\right)$ with $h(F) \neq\{0\}$ and $s_{\circ}=-\nu_{1} / N_{1}=-\nu_{2} / N_{2}$, and if $P$ does not belong to a third component $E_{i}$ of $h^{-1}\left(f^{-1}\{0\}\right)$ with $s_{\circ}=-\nu_{i} / N_{i}$.

Theorem 2.5. Let $f \in \mathbb{C}\left[x_{1}, x_{2}, x_{3}\right] \backslash \mathbb{C}$ and let $s_{\circ} \in \mathbb{Q}$. Fix an embedded resolution $h: X \rightarrow U$ of $f^{-1}\{0\}$ in $\left(\mathbb{A}_{\mathbb{C}}^{3}, 0\right)$ and use the notation of Section 1. Suppose that $s_{\circ}$ is not a pole of $Z_{\mathrm{Hod}}(f, s)$ of order 3 . Then $s_{\circ}$ is a pole of $Z_{\mathrm{Hod}}(f, s)$ of order 2 if and only if

(i) there is a special point for $s_{\circ}$, or

(ii) there exist two components $E_{1}$ and $E_{2}$ of $h^{-1}\left(f^{-1}\{0\}\right)$ satisfying $E_{1} \cap$ $E_{2} \neq \emptyset$ and $s_{\circ}=-\nu_{1} / N_{1}=-\nu_{2} / N_{2}$, and such that the curve $E_{1} \cap E_{2}$ has a non-rational irreducible component $F$ with $h(F)=\{0\}$, or

(iii) there exist two components $E_{1}$ and $E_{2}$ of $h^{-1}\left(f^{-1}\{0\}\right)$ satisfying $E_{1} \cap$ $E_{2} \neq \emptyset$ and $s_{\circ}=-\nu_{1} / N_{1}=-\nu_{2} / N_{2}$, and such that the curve $E_{1} \cap E_{2}$ has a rational irreducible component $F$ with $h(F)=\{0\}$ and intersecting at least three times other components $E_{j}$ of $h^{-1}\left(f^{-1}\{0\}\right)$ with $\nu_{j}+s_{\circ} N_{j} \neq 1$.

Proof. First note that by Proposition 2.3 there do not exist three components $E_{1}, E_{2}$ and $E_{3}$ of $h^{-1}\left(f^{-1}\{0\}\right)$ satisfying $\left(E_{1} \cap E_{2} \cap E_{3}\right) \cap h^{-1}\{0\} \neq \emptyset$ and $s_{\circ}=-\nu_{1} / N_{1}=-\nu_{2} / N_{2}=-\nu_{3} / N_{3}$. Let us look for "the residue of order $2 "$ at $s_{\circ}$. Let $F_{1}, \ldots, F_{r}$ be exactly the irreducible components with $h\left(F_{i}\right)=\{0\}$ of all the curves $E_{\{j, k\}}$ satisfying $s_{\circ}=-\nu_{j} / N_{j}=-\nu_{k} / N_{k}$. 
When $F_{i}$, for $i=1, \ldots, r$, is a component of $E_{\{j, k\}}$, we put $N_{i}:=N_{j} N_{k}$. Suppose that $F_{i}$ intersects $k_{i}$ times other components $E_{j}^{(i)}$ (for $j=1, \ldots, k_{i}$ ) with $\nu_{j}^{(i)}+s_{\circ} N_{j}^{(i)} \neq 1$. If we denote the contribution of $F_{i}$ to the residue of order 2 at $s_{\circ}$ by $\mathcal{R}_{i}$ and if we denote $\nu_{j}^{(i)}+s_{\circ} N_{j}^{(i)}$ by $\alpha_{j}^{(i)}$, then we have

$$
\mathcal{R}_{i}=\frac{(1-u v)^{2}}{N_{i}(u v)^{2 s_{\circ}}}\left(u v-g_{i} u-g_{i} v+\left(1-k_{i}\right)+\sum_{j=1}^{k_{i}} \frac{u v-1}{(u v)^{\alpha_{j}^{(i)}}-1}\right),
$$

for $i \in\{1, \ldots, r\}$, where $g_{i}$ is the genus of $F_{i}$. Let $P_{1}, \ldots, P_{s}$ be exactly the special points for $s_{\circ}$. When $P_{i}$ belongs to the components $E_{j}, E_{k}$ and $E_{l}$ with $s_{\circ}=-\nu_{j} / N_{j}=-\nu_{k} / N_{k}$ and $s_{\circ} \neq-\nu_{l} / N_{l}$, we put $\tilde{N}_{i}:=N_{j} N_{k}$ and $\widetilde{\alpha}_{i}:=\nu_{l}+s_{\circ} N_{l}$. When $P_{i}$ only belongs to the components $E_{j}$ and $E_{k}$ with $s_{\circ}=-\nu_{j} / N_{j}=-\nu_{k} / N_{k}$, we also put $\tilde{N}_{i}:=N_{j} N_{k}$, but now we artificially put $\widetilde{\alpha}_{i}:=1$. If we denote the contribution of $P_{i}$ to the residue of order 2 at $s_{\circ}$ by $\mathcal{Q}_{i}$, then we have for $i \in\{1, \ldots, s\}$ that

$$
\mathcal{Q}_{i}=\frac{(1-u v)^{2}}{\widetilde{N}_{i}(u v)^{2 s_{\circ}}} \frac{u v-1}{(u v)^{\widetilde{\alpha}_{i}}-1} .
$$

For $i \in\{1, \ldots, r\}$, write $k_{i}=k_{i}^{\text {pos }}+k_{i}^{\text {neg }}$, where $k_{i}^{\text {pos }}$ and $k_{i}^{\text {neg }}$ stand for the number of intersections of $F_{i}$ with components $E_{j}^{(i)}$ with $\alpha_{j}^{(i)}>0$ and $\alpha_{j}^{(i)}<0$, respectively; and suppose for convenience that $\alpha_{j}^{(i)}>0$ precisely for $j \in\left\{1, \ldots, k_{i}^{\text {pos }}\right\}$. Also we write $\beta_{j}^{(i)}=-\alpha_{j}^{(i)}$ if $\alpha_{j}^{(i)}<0$. We treat the special points in exactly the same way, now looking at the sign of $\widetilde{\alpha}_{j}$, for $j=1, \ldots, s$. In particular, we have $s=s^{\text {pos }}+s^{\text {neg }}$ and $\widetilde{\alpha}_{j}>0$ precisely for $j \in\left\{1, \ldots, s^{\text {pos }}\right\}$. Note that $\alpha_{j}^{(i)}$ and $\widetilde{\alpha}_{j}$ cannot be zero by the assumptions of the theorem. With this notation we can write

$$
\begin{aligned}
& \mathcal{R}_{i}=\frac{(1-u v)^{2}}{N_{i}(u v)^{2 s_{\circ}}}\left(u v-g_{i} u-g_{i} v+\left(1-k_{i}\right)+\sum_{j=1}^{k_{i}^{\text {pos }}} \frac{u v-1}{(u v)^{\alpha_{j}^{(i)}}-1}\right. \\
& \left.+\sum_{j=k_{i}^{\mathrm{pos}}+1}^{k_{i}} \frac{(u v)^{\beta_{j}^{(i)}}(u v-1)}{1-u v^{\beta_{j}^{(i)}}}\right) \quad \text { for } i=1, \ldots, r, \\
& \mathcal{Q}_{i}=\frac{(1-u v)^{2}}{\widetilde{N}_{i}(u v)^{2 s_{\circ}}} \frac{u v-1}{(u v)^{\widetilde{\alpha}_{i}-1}} \quad \text { for } i=1, \ldots, s^{\mathrm{pos}} \text {, and } \\
& \mathcal{Q}_{i}=\frac{(1-u v)^{2}}{\widetilde{N}_{i}(u v)^{2 s_{\circ}}} \frac{(u v)^{\widetilde{\beta}_{i}}(u v-1)}{1-(u v)^{\widetilde{\beta}_{i}}} \quad \text { for } i=s^{\mathrm{pos}}+1, \ldots, s
\end{aligned}
$$


and $s_{\circ}$ is a pole of order 2 if and only if $\sum_{i=1}^{r} \mathcal{R}_{i}+\sum_{i=1}^{s} \mathcal{Q}_{i} \neq 0$, or equivalently

$$
T:=\frac{(u v)^{2 s_{\circ}}}{(1-u v)^{2}}\left(\sum_{i=1}^{r} \mathcal{R}_{i}+\sum_{i=1}^{s} \mathcal{Q}_{i}\right) \neq 0 .
$$

Suppose first that there are no special points for $s_{\circ}$, that all the curves $F_{i}$ are rational and that $k_{i} \leq 2$ for all $i \in\{1, \ldots, r\}$. Note that $k_{i} \geq 1$ by Lemma 1.2 .1 , since $F_{i}$ is a rational curve. But when $k_{i}=1$ or $k_{i}=2$ it easily follows from the relation of Lemma 1.2.1 that $\mathcal{R}_{i}=0$.

We now still have to prove that whenever one of the three conditions is fulfilled, the rational number $s_{\circ}$ will be a pole of order 2. First assume that condition (ii) is fulfilled and suppose for example that $g_{1}=g\left(F_{1}\right)>0$. Because

$$
\lim _{u \rightarrow 0} T=\sum_{i=1}^{r} \frac{1-k_{i}^{\text {neg }}-g_{i} v}{N_{i}}+\sum_{i=1}^{s^{\mathrm{pos}}} \frac{1}{\widetilde{N}_{i}} \neq 0,
$$

since $g_{1}>0$ and $g_{i} \geq 0$ for $i \in\{2, \ldots, r\}$, we see that $T \neq 0$. So $s_{\circ}$ is a pole of order 2 in this case.

From now on we assume that all the curves $F_{i}$ are rational, so condition (ii) cannot be fulfilled anymore. In fact, for computing the residue, we may also assume that all the rational curves $F_{i}$ have $k_{i} \geq 3$. Let us now treat the case that condition (i) is satisfied, i.e., there are special points for $s_{\circ}$, but condition (iii) is not, i.e., $r=0$. Then we have

$$
\lim _{u \rightarrow 0} T=\sum_{i=1}^{s^{\mathrm{pos}}} \frac{1}{\widetilde{N}_{i}}
$$

which can only be zero if $s^{\text {pos }}=0$. In this case

$$
T=\sum_{i=1}^{s} \frac{(u v)^{\widetilde{\beta}_{i}}(u v-1)}{\widetilde{N}_{i}\left(1-(u v)^{\widetilde{\beta}_{i}}\right)}
$$

and we can consider $q:=\min \left\{\widetilde{\beta}_{i} \mid 1 \leq i \leq s\right\}$. Then clearly

$$
\lim _{u \rightarrow 0}\left(\frac{T}{(u v)^{q}}\right)=-\sum \frac{1}{\widetilde{N}_{i}} \neq 0
$$

where the summation runs over $i \in\{1, \ldots, s\}$ with $\widetilde{\beta}_{i}=q$. This implies that $T \neq 0$ and hence that $s_{\circ}$ is a pole of order 2 . 
Finally we suppose that condition (iii) is satisfied, i.e., $r \geq 1$. We have to show that $T \neq 0$. So suppose that $\lim _{u \rightarrow 0} T=0$, otherwise we are done. Because $g_{i}=0$ for each $i \in\{1, \ldots, r\}$, we have

$$
\sum_{i=1}^{r} \frac{1-k_{i}^{\mathrm{neg}}}{N_{i}}+\sum_{i=1}^{s^{\mathrm{pos}}} \frac{1}{\widetilde{N}_{i}}=0 .
$$

This equality implies that $T=\sum_{i=1}^{r} R_{i}+\sum_{i=1}^{s} Q_{i}$, where

$$
\begin{gathered}
R_{i}=\frac{1}{N_{i}}\left(u v+\sum_{j=1}^{k_{i}^{\mathrm{pos}}} \frac{u v-(u v)^{\alpha_{j}^{(i)}}}{(u v)^{\alpha_{j}^{(i)}}-1}+\sum_{j=k_{i}^{\mathrm{pos}}+1}^{k_{i}} \frac{(u v)^{\beta_{j}^{(i)}}(u v-1)}{1-(u v)^{\beta_{j}^{(i)}}}\right), \\
Q_{i}=\frac{u v-(u v)^{\widetilde{\alpha}_{i}}}{\widetilde{N}_{i}\left((u v)^{\widetilde{\alpha}_{i}}-1\right)} \quad \text { for } i=1, \ldots, s^{\mathrm{pos}}, \text { and } \\
Q_{i}=\frac{(u v)^{\widetilde{\beta}_{i}}(u v-1)}{\widetilde{N}_{i}\left(1-(u v)^{\widetilde{\beta}_{i}}\right)} \quad \text { for } i=s^{\mathrm{pos}}+1, \ldots, s .
\end{gathered}
$$

For simplicity we can assume that $\widetilde{\alpha}_{i} \neq 1$ for $i \in\left\{1, \ldots, s^{\text {pos }}\right\}$. Now we distinguish two possibilities.

(1) Suppose that none of the $\beta_{j}^{(i)}$ and $\widetilde{\beta}_{i}$ equals 1 , that $k_{i}^{\text {pos }}=1$ for $i \in\{1, \ldots, r\}$ and that $s^{\mathrm{pos}}=0$. In this case we have

$$
T=\sum_{i=1}^{r} \frac{1}{N_{i}}\left(\frac{(u v)^{\alpha_{1}^{(i)}}(u v-1)}{(u v)^{\alpha_{1}^{(i)}}-1}+\sum_{j=2}^{k_{i}} \frac{(u v)^{\beta_{j}^{(i)}}(u v-1)}{1-(u v)^{\beta_{j}^{(i)}}}\right)+\sum_{i=1}^{s} \frac{(u v)^{\widetilde{\beta}_{i}}(u v-1)}{\widetilde{N}_{i}\left(1-(u v)^{\widetilde{\beta}_{i}}\right)} .
$$

Denote by $q$ the minimum of the set $\left\{\left|\alpha_{j}^{(i)}\right| \mid 1 \leq i \leq r, 1 \leq j \leq k_{i}\right\} \cup\left\{\widetilde{\beta}_{i} \mid\right.$ $1 \leq i \leq s\}$. Now fix $i \in\{1, \ldots, r\}$. By Lemma 1.2.1 we know that

$$
\alpha_{1}^{(i)}=\left(k_{i}-2\right)+\sum_{j=2}^{k_{i}} \beta_{j}^{(i)}>\sum_{j=2}^{k_{i}} \beta_{j}^{(i)}
$$

which implies that $\alpha_{1}^{(i)}>\beta_{j}^{(i)}$ for $j=2, \ldots, k_{i}$. We obtain that

$$
\begin{aligned}
\lim _{u \rightarrow 0}\left(\frac{T}{(u v)^{q}}\right)= & -\sum_{i=1}^{r} \frac{1}{N_{i}} \#\left\{j \mid 2 \leq j \leq k_{i} \text { and } \beta_{j}^{(i)}=q\right\} \\
& -\sum_{i=1}^{s} \frac{1}{\widetilde{N}_{i}} \delta\left(\widetilde{\beta}_{i}=q\right)<0
\end{aligned}
$$


where $\delta\left(\widetilde{\beta}_{i}=q\right)=1$ if $\widetilde{\beta}_{i}=q$ and $\delta\left(\widetilde{\beta}_{i}=q\right)=0$ otherwise. This yields that $T \neq 0$.

(2) Suppose exactly the negation of (1).

Denote by $q_{1}$ the minimum of the set $\left\{\left|\alpha_{j}^{(i)}\right| \mid 1 \leq i \leq r, 1 \leq j \leq k_{i}\right\} \cup\left\{\left|\widetilde{\alpha}_{i}\right| \mid\right.$ $1 \leq i \leq s\} \cup\{1\}$.

(i) $\mathbf{q}_{\mathbf{1}}=\mathbf{1}$. We see that

$$
\begin{aligned}
\lim _{u \rightarrow 0}\left(\frac{T}{(u v)}\right)= & \sum_{i=1}^{r} \frac{1}{N_{i}}\left(1-k_{i}^{\mathrm{pos}}-\#\left\{j \mid k_{i}^{\mathrm{pos}}+1 \leq j \leq k_{i} \text { and } \beta_{j}^{(i)}=1\right\}\right) \\
& -\sum_{i=1}^{s^{\mathrm{pos}}} \frac{1}{\widetilde{N}_{i}}-\sum_{i=s^{\mathrm{pos}}+1}^{s} \frac{1}{\widetilde{N}_{i}} \delta\left(\widetilde{\beta}_{i}=1\right) .
\end{aligned}
$$

By Lemma 1.2.1 we know that $\sum_{j=1}^{k_{i}} \alpha_{j}^{(i)}=k_{i}-2$. Because $k_{i} \geq 3$, we find that $k_{i}^{\text {pos }} \geq 1$ for each $i \in\{1, \ldots, r\}$. So every term in the sum above is nonpositive. But the assumption in (2) precisely implies that at least one term, and therefore also the whole sum, is strictly negative. So we can conclude that $T \neq 0$.

(ii) $\mathbf{q}_{\mathbf{1}}<\mathbf{1}$. Now we can start an analogous process as in the last and most difficult part of the proof of [12, Theorem 3.4]. Let us recall this process very briefly. Supposing that $\lim _{u \rightarrow 0}\left(T /(u v)^{q_{1}}\right)=0$ will imply that in the expressions for $R_{i}$ and $Q_{i}$ we may as well omit the terms of the form $\left(u v-(u v)^{\alpha}\right) /\left((u v)^{\alpha}-1\right)$ with $\alpha=q_{1}$ and replace the terms of the form $(u v)^{\beta}(u v-1) /\left(1-(u v)^{\beta}\right)$ with $\beta=q_{1}$ by $-u v$. Then the minimum of the remaining exponents of $u v$ in the expression for $T$ will be strictly larger than $q_{1}$. Denote it by $q_{2}$ and suppose that also $q_{2}<1$. When $\lim _{u \rightarrow 0}\left(T /(u v)^{q_{2}}\right)=0$, we can perform the same cancellation ritual. After a finite number of steps this process will clearly stop. When it stops at step $n$, then either $q_{n}=1$, or $q_{n}<1$ and $\lim _{u \rightarrow 0}\left(T /(u v)^{q_{n}}\right) \neq 0$. Now it is remarkable that when $q_{n}=1$ the expression for $\lim _{u \rightarrow 0}(T /(u v))$ is precisely equal to the one in case (i), hence also here this limit is different from zero. This ends the proof of Theorem 2.5.

Now we will give the statement that embraces the poles of order 2 as well as the poles of order 3 .

TheOREm 2.6. Let $f \in \mathbb{C}\left[x_{1}, x_{2}, x_{3}\right] \backslash \mathbb{C}$ and let $s_{\circ} \in \mathbb{Q}$. Fix a good embedded resolution $h: X \rightarrow U$ of $f^{-1}\{0\}$ in $\left(\mathbb{A}_{\mathbb{C}}^{3}, 0\right)$ and use the notation of 
Section 1. Let $F_{1}, \ldots, F_{r}$ be exactly the irreducible components with $h\left(F_{i}\right)=$ $\{0\}$ of all the curves $E_{\{j, k\}}$ satisfying $s_{\circ}=-\nu_{j} / N_{j}=-\nu_{k} / N_{k}$. Then $s_{\circ}$ is a pole of $Z_{\mathrm{Hod}}(f, s)$ of order 2 or 3 if and only if

(i) there is a special point for $s_{\circ}$, or

(ii) there is a non-rational curve $F_{i}$, or

(iii) there is a rational curve $F_{i}$ intersecting at least three times other components $E_{j}$ with $\nu_{j}+s_{\circ} N_{j} \neq 1$, or

(iv) there is a cycle of rational curves $F_{i}$.

See (1.2) for the notion of good embedded resolution and Definition 2.4.1 for the notion of special point.

Proof. Suppose that $s_{\circ}$ is a pole of order 3. Then there exist three components $E_{1}, E_{2}$ and $E_{3}$ of $h^{-1}\left(f^{-1}\{0\}\right)$ satisfying $\left(E_{1} \cap E_{2} \cap E_{3}\right) \cap$ $h^{-1}\{0\} \neq \emptyset$ and $s_{\circ}=-\nu_{1} / N_{1}=-\nu_{2} / N_{2}=-\nu_{3} / N_{3}$. Since $h$ is a good embedded resolution, at least one of these three components is entirely mapped by $h$ onto the origin of $\mathbb{A}_{\mathbb{C}}^{3}$. Suppose for example that $h\left(E_{1}\right)=\{0\}$. Then we have on the surface $E_{1}$ two intersecting curves $F_{1}$ and $F_{2}$, both satisfying the relation of Lemma 1.2.1. Suppose now that conditions (i), (ii) and (iv) are not fulfilled. Then, by repeatedly using Lemma 1.2.1, we can prove in exactly the same way as in the first part of the proof of $[12$, Theorem 3.4] that condition (iii) should be satisfied. This fact, together with Theorem 2.5, already implies that at least one of the four conditions is satisfied whenever $s_{\circ}$ is a pole of order 2 or 3 .

Now we pass on to the other implication. First observe that condition (iv) always implies that $s_{\circ}$ is a pole of order 3 . So suppose that condition (i), (ii) or (iii) is satisfied and that $s_{\circ}$ is not a pole of order 3. Then Theorem 2.5 yields that $s_{\circ}$ is a pole of order 2 .

Remarks 2.7. (i) Let $s_{\circ} \in \mathbb{Q}$ be a pole of $Z_{\text {Hod }}(f, s)$ of order 3 and suppose that conditions (i), (ii) and (iii) are not satisfied. Then we know by the previous theorem that there should be a cycle of rational curves $F_{i}$. But actually, one can even easily show that this cycle can be taken to be a closed subset of one elected exceptional surface.

(ii) When 0 is an isolated singular point of $f^{-1}\{0\}$, we say that the good embedded resolution $h: X \rightarrow U$ of $f^{-1}\{0\}$ in $\left(\mathbb{A}_{\mathbb{C}}^{3}, 0\right)$ is a fair resolution if $h\left(E_{i}\right)=\{0\}$ for each exceptional surface $E_{i}$. Note that such a fair resolution always exists when the origin is an isolated singularity of $f^{-1}\{0\}$. 
Let $f \in \mathbb{C}\left[x_{1}, x_{2}, x_{3}\right] \backslash \mathbb{C}$ such that $f^{-1}\{0\}$ has an isolated singularity at 0 and suppose that $h: X \rightarrow U$ is a fair resolution. Then one easily sees that there cannot be special points for $s_{\circ}$; so in the statement of Theorem 2.6 we can simply omit condition (i).

2.8. Let us now consider the general situation of a polynomial in $n$ variables. Also in this case we can introduce the notion of a special point for a rational number $s_{\circ}$. This will be a point $P \in h^{-1}\{0\}$ which belongs to exactly $n-1$ irreducible components $E_{i}$ of $h^{-1}\left(f^{-1}\{0\}\right)$ satisfying $s_{\circ}=$ $-\nu_{i} / N_{i}$, but which does not belong to any of the curves $F_{i}$ in the statement below. We leave the details to the reader.

Theorem 2.8.1. Let $n \in \mathbb{N}_{\geq 3}, f \in \mathbb{C}\left[x_{1}, \ldots, x_{n}\right] \backslash \mathbb{C}$ and $s_{\circ} \in \mathbb{Q}$. Fix a good embedded resolution $h: X \rightarrow U$ of $f^{-1}\{0\}$ in $\left(\mathbb{A}_{\mathbb{C}}^{n}, 0\right)$ and use the notation of Section 1 . Let $F_{1}, \ldots, F_{r}$ be exactly the irreducible components with $h\left(F_{i}\right)=\{0\}$ of all the curves $E_{I}$ satisfying $s_{\circ}=-\nu_{i} / N_{i}$ for $i \in I$, where $I \subseteq S$ with $|I|=n-1$. Then $s_{\circ}$ is a pole of $Z_{\mathrm{Hod}}(f, s)$ of order $n-1$ or $n$ if and only if

(i) there is a special point for $s_{\circ}$, or

(ii) there is a non-rational curve $F_{i}$, or

(iii) there is a rational curve $F_{i}$ intersecting at least three times other components $E_{j}$ with $\nu_{j}+s_{\circ} N_{j} \neq 1$, or

(iv) there is a cycle of rational curves $F_{i}$.

Remark 2.8.2. When $n=2$, we can interpret a special point as an intersection point of an irreducible component $E_{i}$ of the strict transform of $f^{-1}\{0\}$ satisfying $s_{\circ}=-\nu_{i} / N_{i}$ with an exceptional curve $E_{j}$ satisfying $s_{\circ} \neq-\nu_{j} / N_{j}$. With this interpretation Theorem 2.8.1 still holds in the case of curves, except when the germ $(f, 0)$ is analytically equivalent to $\left(x_{1}^{N} x_{2}^{N}, 0\right)$ for some $N \in \mathbb{N} \backslash\{0\}$. Note that in this exceptional case the germ of $\left(f^{-1}\{0\}\right)_{\text {red }}$ at 0 is analytically equivalent to a node. The proof is exactly the same as for arbitrary $n$, except the part where we integrate the poles of order 2 in the statement of the poles of order 1 ; in particular it might happen that $s_{\circ}$ is a pole of order 2 , but yet none of the four conditions is satisfied. Now, using [14, Section 3], it only needs an easy verification to see that this problem can only occur when the germ $(f, 0)$ is analytically equivalent to $\left(x_{1}^{N} x_{2}^{N}, 0\right)$ for some $N \in \mathbb{N} \backslash\{0\}$.

Also note that conditions (ii) and (iv) of Theorem 2.8.1 cannot occur in this situation of curves. Then Theorem 2.8.1, omitting conditions (ii) and 
(iv), is equivalent to the analogue for $Z_{\text {Hod }}(f, s)$ of the theorem for $Z_{\text {top }}(f, s)$ of Veys, recall Theorem 0.3 .1 and its succeeding remark concerning the condition of intersecting at least three times other components.

\section{$\S 3$. The motivic zeta function}

3.1. Let us sketch very briefly the notion of a pole of the motivic zeta function $Z_{\text {mot }}(f, s)$. For this, we start by representing $Z_{\text {mot }}(f, s)$ as a quotient of two polynomials in the variable $\mathbb{L}^{-s}$ over a "well-chosen" ring $A$, say for example $Z_{\text {mot }}(f, s)=N\left(\mathbb{L}^{-s}\right) / D\left(\mathbb{L}^{-s}\right)$. The main point about $A$ is that in this ring the elements $1-\mathbb{L}^{q}$ and $b \mathbb{L}^{a}$, for $q \in \mathbb{Q} \backslash\{0\}, b \in \mathbb{Z} \backslash\{0\}$ and $a \in \mathbb{Q}$, are units. For the rigorous definition of $A$ one first introduces the ring

$$
\mathcal{M}_{\mathbb{C}}^{\mathbb{Q}}:=\frac{\mathcal{M}_{\mathbb{C}}\left[\left(T_{i}\right)_{i \in \mathbb{N} \backslash\{0\}}\right]}{\left(T_{i}^{i}-\mathbb{L}, T_{k}^{j}-T_{m}^{l}\left(\frac{j}{k}=\frac{l}{m}\right)\right)},
$$

where the denominator stands for the ideal generated by the elements $T_{i}^{i}-\mathbb{L}$ and $T_{k}^{j}-T_{m}^{l}$, for $i, j, k, l, m \in \mathbb{N} \backslash\{0\}$ and $j / k=l / m$. Intuitively we just allow ourselves to work also with rational powers of $\mathbb{L}$. With some abuse of notation we will write $\mathbb{L}^{j / k}$ for the class of $T_{k}^{j}$ in $\mathcal{M}_{\mathbb{C}}^{\mathbb{Q}}$. Then $A$ is defined to be the localization of $\mathcal{M}_{\mathbb{C}}^{\mathbb{Q}}$ with respect to the elements $1-\mathbb{L}^{q}$ and $b$, for $q \in \mathbb{Q} \backslash\{0\}$ and $b \in \mathbb{Z} \backslash\{0\}$. For a nonzero polynomial $H\left(\mathbb{L}^{-s}\right)$ over $A$ and a rational number $q$ we denote by $n(H, q)$ the unique natural number such that $\left(\mathbb{L}^{-s}-\mathbb{L}^{-q}\right)^{n(H, q)} \mid H\left(\mathbb{L}^{-s}\right)$ and $\left(\mathbb{L}^{-s}-\mathbb{L}^{-q}\right)^{n(H, q)+1} \nmid H\left(\mathbb{L}^{-s}\right)$ in $A\left[\mathbb{L}^{-s}\right]$. Now we simply say that a rational number $q$ is a pole of $Z_{\operatorname{mot}}(f, s)$ if $n(D, q)>n(N, q)$. If $q$ is a pole of $Z_{\text {mot }}(f, s)$, then we call $n(D, q)-n(N, q)$ the order of the pole $q$. For more details we refer to [12, Section 4].

3.2. Theorem 2.8.1 also holds for the motivic zeta function! Indeed, the implication which states that $s_{\circ}$ is a pole of order $n-1$ or $n$ if at least one of the four conditions is satisfied, easily follows from the technique of specialization. When we return for example to the proof of Theorem 2.5, we obtain by an easy calculation that $s_{\circ}$ is a pole of $Z_{\operatorname{mot}}(f, s)$ of order 2 if and only if

$$
\sum_{i=1}^{r} \frac{(\mathbb{L}-1)^{2}}{N_{i} \mathbb{L}^{2 s_{\circ}}}\left(\left[F_{i}^{\circ}\right]+\sum_{j=1}^{k_{i}} \frac{(\mathbb{L}-1) \mathbb{L}^{-\alpha_{j}^{(i)}}}{1-\mathbb{L}^{-\alpha_{j}^{(i)}}}\right)+\sum_{i=1}^{s} \frac{(\mathbb{L}-1)^{2}}{\widetilde{N}_{i} \mathbb{L}^{2 s_{\circ}}} \frac{(\mathbb{L}-1) \mathbb{L}^{-\widetilde{\alpha}_{i}}}{1-\mathbb{L}^{-\widetilde{\alpha}_{i}}} \neq 0
$$

in $A$; see [12, Proposition 5.4.1] for a very similar fact (and proof). But now we note that "the residue of order 2 " at $s_{\circ}$ of the Hodge zeta function 
$Z_{\text {Hod }}(f, s)$ can obviously be seen as the specialization of the expression above. Thus, when $s_{\circ}$ is a pole of $Z_{\mathrm{Hod}}(f, s)$ of order 2 , the same is true for $Z_{\text {mot }}(f, s)$.

For the other implication we only have to check, except for some other trivial remarks, that

$$
\left[F_{i}^{\circ}\right]+\sum_{j=1}^{k_{i}} \frac{(\mathbb{L}-1) \mathbb{L}^{-\alpha_{j}^{(i)}}}{1-\mathbb{L}^{-\alpha_{j}^{(i)}}}=0
$$

in $A$, for each $i \in\{1, \ldots, r\}$ such that $F_{i}$ is a rational curve with $k_{i}=1$ or 2 . Recall that $k_{i}$ stands for the number of times that $F_{i}$ intersects other components $E_{j}^{(i)}$ with $\nu_{j}^{(i)}+s_{\circ} N_{j}^{(i)} \neq 1$. Again, this vanishing fact will easily follow from Lemma 1.2.1.

\section{REFERENCES}

[1] N. A'Campo, La fonction zeta d'une monodromie, Comment. Math. Helv., 50 (1975), 233-248.

[2] E. Artal, P. Cassou-Noguès, I. Luengo, and A. Melle, Monodromy Conjecture for some surface singularities, Ann. Sci. École Norm. Sup., 35.4 (2002), 605-640.

[3] J. Denef and F. Loeser, Caractéristiques d'Euler-Poincaré, fonctions zeta locales, et modifications analytiques, J. Amer. Math. Soc., 5 (1992), 705-720.

[4] - Motivic Igusa zeta functions, J. Algebraic Geom., 7 (1998), 505-537.

[5] H. Hironaka, Resolution of an algebraic variety over a field of characteristic zero, Ann. of Math., 79 (1964), 109-326.

[6] J. Kollár, Singularities of Pairs, Summer Research Institute on Algebraic Geometry (Santa Cruz 1995), Proceedings of Symposia in Pure Mathematics, 62.1 (Amer. Math. Soc., 1997), 221-287.

[7] F. Loeser, Fonctions d'Igusa p-adiques et polynômes de Bernstein, Amer. J. Math., 110 (1988), 1-22.

[8] —. Fonctions d'Igusa p-adiques, polynômes de Bernstein, et polyèdres de Newton, J. Reine Angew. Math., 412 (1990), 75-96.

[9] B. Poonen, The Grothendieck ring of varieties is not a domain, Math. Res. Letters, 9.4 (2002), 493-498.

[10] B. Rodrigues, On the Monodromy Conjecture for curves on normal surfaces, Math. Proc. Cambridge Philos. Soc., 136 (2004), 313-324.

[11] B. Rodrigues and W. Veys, Holomorphy of Igusa's and topological zeta functions for homogeneous polynomials, Pacific J. Math., 201.2 (2001), 429-440.

[12] — Poles of zeta functions on normal surfaces, Proc. London Math. Soc., 87 (2003), 164-196.

[13] W. Veys, Poles of Igusa's local zeta function and monodromy, Bull. Soc. Math. France, 121 (1993), 545-598. 
[14] Determination of the poles of the topological zeta function for curves, Manuscripta Math., 87 (1995), 435-448.

[15] - More congruences for numerical data of an embedded resolution, Compositio Math., 112 (1998), 313-331.

[16] The topological zeta function associated to a function on a normal surface germ, Topology, 38 (1999), 439-456.

K. U. Leuven

Departement Wiskunde

Celestijnenlaan 200B

3001 Leuven

Belgium

bart.rodrigues@wis. kuleuven.ac. be 